\title{
Avaliação do Processo de Aconselhamento Vocacional: Revisão da Literatura ${ }^{1}$
}

\author{
Liliana da Costa Faria ${ }^{2}$ \\ Maria do Céu Taveira \\ Universidade do Minho
}

\begin{abstract}
RESUMO - As questões acerca do processo de intervenção têm sido cada vez mais alvo de atenção e estudo na Psicologia. Este artigo apresenta uma revisão da literatura acerca da panorâmica atual do processo de aconselhamento vocacional. Deste modo, são abordados, uma descrição do processo de aconselhamento vocacional e das variáveis comuns ao aconselhamento psicológico pessoal e vocacional, seguindo-se uma breve caracterização das investigações sobre o processo da intervenção psicológica vocacional. Por último, apresenta-se um conjunto de sugestões para futuras pesquisas.
\end{abstract}

Palavras-chave: Aconselhamento vocacional; Processo de aconselhamento; Avaliação.

\section{Evaluation of the Process of Career Counseling: A Literature Review}

\begin{abstract}
Questions concerning the intervention process have been increasingly target of attention and research within Psychology. This article presents a review of the literature related to the process of career counseling. In this context, the process of career counseling, and the common characteristics of career and personal counseling are analyzed. Additionally, a brief characterization is presented of the main research studies of the career counseling process. Finally, suggestions for future research are outlined.
\end{abstract}

Keywords: Career counselling; Career counselling process; Evaluation

Ao longo da história da investigação do processo de aconselhamento psicológico vocacional, dois dos temas com desenvolvimento mais interessante foram: a relação entre os processos de aconselhamento psicológico vocacional e pessoal e, a relação entre os resultados e o processo do aconselhamento psicológico vocacional (Heppner \& Heppner, 2007; Heppner \& Jordan, 2007; Whiston, Lindeman, Rahardja, \& Reed, 2005).

Com efeito, a investigação do processo de aconselhamento vocacional inspirou-se no estudo prévio do processo do aconselhamento pessoal (Heppner, \& Heppner, 2004). Em 1938, Frank Robinson ensinava um practicum em aconselhamento psicológico a seniores e estudantes graduados da sua faculdade, gravando as sessões de intervenção dos seus alunos psicólogos, para posterior visualização. Com este método, ao longo de cerca de dez anos, foi sendo recolhido e gravado um conjunto valioso de dados para análise do processo de aconselhamento. Com base nesse material, Robinson (1950) definiu categorias do comportamento do psicólogo e do cliente, no contexto do aconselhamento pessoal, quais sejam, o silêncio, a reflexão, a tentativa de análise e a interpretação (Porter, 1943). Os resultados das suas análises indicam que as observações do psicólogo em cada uma das sessões tinham impacto nos comportamentos posteriores, quer do cliente, quer do psicólogo (Robinson, 1950).

1 Trabalho realizado no âmbito de um projecto de doutoramento, financiado pela bolsa SFRH/BD/18637/2004 da Fundação para a Ciência e a Tecnologia (FCT).

2 Endereço para correspondência: Av. da Igreja, 291. 4750-723 Tamel S. Veríssimo. Barcelos, Portugal. Fone: 917165417.E-mail.lilianafaria@ delphis.com.pt
Este trabalho deu origem, nos anos 1960 e 1970, a um elevado crescimento do número de pesquisas acerca do processo de aconselhamento psicológico pessoal, bem como a um aumento do interesse pelo estudo das especificidades do processo do aconselhamento psicológico vocacional. Todavia, a partir dos anos 1970, diversos investigadores apresentam uma visão negativa do que seria o futuro da investigação do processo da intervenção psicológica. Malan (1973) escreve, sobre este propósito, que os investigadores se sentem desiludidos e menos interessados pela pesquisa do processo da intervenção. Goldman (1976), por sua vez, critica a maioria dos estudos, pela sua natureza microscópica, limitada, e análoga, responsabilizando as instituições públicas pela proliferação de estudos triviais no domínio. Este pessimismo expresso em relação ao estudo do aconselhamento psicológico, aliado à convicção arcaica que o aconselhamento psicológico vocacional não envolve uma interacção de natureza especializada, contribuiu para que, nessa época, a investigação do processo de aconselhamento vocacional fosse também diminuindo progressivamente.

Apenas nos anos 1990, isto é, quase vinte anos depois, surge um interesse renovado pela problemática. Nesta época, vários autores procuraram defender a natureza psicológica do aconselhamento vocacional, a partir de definições que fazem menção explícita aos seus elementos cognitivos e emocionais (Blustein \& Spengler, 1995; Gysbers, 2004; Gysbers, Heppner, \& Johnston, 2003). Este fato desperta novamente o interesse de investigar esta modalidade de intervenção e compreender os aspectos psicológicos da mudança, no âmbito da carreira.

Este interesse é, no entanto, rapidamente criticado pelos autores que se dedicam a estudar os resultados do acon- 
selhamento psicológico vocacional e que defendem que a investigação desta modalidade de intervenção deve centrar-se nos mecanismos específicos que produzem os resultados da intervenção, uma vez que são estes que justificam a existência de aconselhamento vocacional e contribuem para a sua validação como prática científica.

Para além desta argumentação, a linha de estudo dos resultados da intervenção vocacional demonstrou, consistentemente, que os psicólogos vocacionais eram eficazes nas suas intervenções, para uma maioria de pessoas (Holland, Magoon, \& Spokane, 1981; Swanson, 1995). Esta constatação levou a um aumento de confiança neste tipo de intervenção psicológica mas, ao mesmo tempo, a uma diminuição do interesse pelo processo da intervenção psicológica vocacional. Desenvolveu-se, assim, uma percepção geral que a maioria das necessidades dos clientes do aconselhamento vocacional estava atendida com sucesso, pelo que não havia necessidade de explorar os mecanismos reais da mudança relacionada com a mesma (Heppner \& Heppner, 2003; Heppner \& Heppner, 2007).

Outro fator de desinteresse prende-se com a modesta contribuição dada a este domínio da literatura vocacional, pelos estudos do processo, quando comparada com a forte contribuição dada pelos estudos dos resultados do aconselhamento. Por exemplo, num estudo sobre o processo da intervenção psicológica vocacional, Krischner, Hoffman e Hill (1994) verificaram que os componentes mais úteis do processo da intervenção eram a introspecção e o desafio, indicando que o cliente é beneficiado pela aprendizagem sobre si próprio e sobre o mundo ocupacional. Embora esta informação seja útil para a intervenção, torna-se pouco vantajoso replicar este estudo com amostras de maiores dimensões, dado o elevado investimento de tempo e energia envolvidos na coleta de dados, e os contributos reduzidos que tal estudo traria para o desenvolvimento da tecnologia daquela modalidade de intervenção.

Ainda assim, em termos gerais, os investigadores dos resultados do aconselhamento vocacional foram compreendendo que a separação entre o processo e os resultados era artificial, uma vez que o estudo dos resultados apenas incidia sobre a eficácia da intervenção, ignorando o porquê desses resultados. Ao mesmo tempo, os investigadores do processo passaram a valorizar a importância do estudo dos resultados, para validar a importância do estudo do processo (Whiston \& cols., 2005).

\section{Aconselhamento psicológico vocacional e pessoal: fatores comuns}

As variáveis do processo que contribuem para a eficácia do aconselhamento psicológico em geral, incluindo o aconselhamento vocacional, têm sido alvo de diversos estudos, ao longo das últimas quatro décadas (Gurman, 1977; Orlinsky \& Howard, 1986; Patterson, 1974). Nesta linha, foram identificados quatro elementos necessários à ocorrência de mudança fruto da intervenção e à sua eficácia. São eles: (1) a qualidade da relação terapêutica; (2) o insight cognitivo; (3) a experiência afetiva e; (4) as expectativas do cliente (Heppner \& Heppner, 2003).
O primeiro desses elementos, a relação terapêutica, foi considerado um dos fatores mais importantes, na literatura do processo do aconselhamento pessoal e vocacional (Hartley \& Strupp, 1983). Gurman (1977) reviu 26 estudos de aconselhamento psicológico pessoal, conduzidos entre 1954 e 1974, e observou uma correlação positiva entre a percepção dos clientes de existência de uma relação terapêutica e de resultados positivos da intervenção. Por sua vez, Orlinsky e Howard (1986), referem a existência de uma correlação positiva entre uma relação terapêutica do tipo colaborativo e resultados positivos da intervenção, ou seja, as intervenções em que o psicólogo encoraja a independência do cliente, estão associadas a resultados desejáveis de intervenção. Deste modo, pode-se afirmar que o papel da relação colaborativa entre psicólogo e clientes é um facilitador de resultados de intervenção de sucesso. Neste sentido, a relação terapêutica foi apontada como a responsável por 30\% a 50\% da variação dos resultados terapêuticos no aconselhamento psicológico pessoal (Gelso \& Cárter, 1994; Horvath \& Luborsky, 1993).

A investigação conduzida até o momento sobre a relação terapêutica no aconselhamento psicológico vocacional tem produzido resultados controversos. Gold, Kivlighan, Kerr e Kramer (1993) e Kirschner e cols. (1994), por exemplo, identificaram componentes afetivos do psicólogo que explicam a mudança na identidade vocacional de um cliente e membros de um grupo. Similarmente, Heppner e Hendricks (1995) verificaram que tanto os clientes indecisos quanto os clientes decididos, auto-relataram a relação terapêutica como sendo importante para o resultado do aconselhamento psicológico vocacional. No entanto, Heppner, Multon, Gysbers, Ellis e Zook (1998), num estudo acerca do processo e dos resultados do aconselhamento vocacional, verificaram que a importância atribuída à relação terapêutica tinha um impacto reduzido ou mesmo nulo, nos resultados da intervenção. Heppner e cols. (1998), não registaram nenhuma correlação linear significativa entre a relação terapêutica e a mudança positiva nas variáveis de resultado. Mais recentemente, Multon e cols. (2001, 2007), registaram resultados divergentes, ao verificar a existência de uma correlação linear significativa entre a percepção dos clientes acerca da existência de uma relação terapêutica ao longo das sessões e as variáveis de resultado alcançados com a intervenção.

Apesar da falta de consenso entre os estudos da relação terapêutica no aconselhamento psicológico vocacional, esta foi apontada como responsável de $1 \%$ a $12 \%$ pelos seus resultados (Heppner \& Heppner, 2003), reforçando a conclusão que os resultados do aconselhamento psicológico vocacional podem ser explicados pela qualidade da relação terapêutica (Gunderson, Najavits, Leonhard, Sullivan, \& Sabo, 1997; Kivlighan \& Shaughnessy, 1995).

O insight cognitivo, ou a crença dos clientes que a aquisição de uma nova percepção sobre os seus próprios problemas pode ajudá-los a resolver esses mesmos problemas, é a segunda variável do processo que concorre para a eficácia do aconselhamento psicológico pessoal e vocacional (Walborn, 1996).

Já na década de 1950 Bergman (1951) referia que os psicólogos podem oferecer insight cognitivo aos clientes de modo diretivo ou não diretivo. O primeiro envolve o fornecimento direto de informação ao cliente, enquanto 
o segundo diz respeito, por exemplo, à reflexão de sentimentos com o cliente. Ambos os tipos de insight estão significativamente relacionados com resultados positivos da intervenção. Outras investigações se seguiram neste âmbito. Assim, por exemplo, num estudo de caso relevante neste âmbito, Hill, Cárter e O'Farrell (1983) verificaram que os estilos interpretativo, confrontativo e experiencial do psicólogo provocaram no cliente um aumento da capacidade de introspecção e de experimentação, bem como uma diminuição da quantidade de tempo gasta na descrição dos seus problemas, dentro e através das sessões. Segundo Hill e cols. (1983), os mecanismos de mudança no aconselhamento parecem estar relacionados com o insigth cognitivo providenciado ao cliente. As medidas de resultado indicam que a intervenção foi positiva e conduziu a uma melhoria significativa do cliente após 12 sessões, bem como uma manutenção desta melhoria, dois meses após a intervenção.

Esta linha de estudo, apesar de escassa, tem permitido esclarecer os fatores comuns e as especificidades dos processos de intervenção psicológica vocacional e pessoal (Bedi, 2004; Gelso \& Carter, 1985; Lewis, 2001).

O terceiro elemento comum ao aconselhamento psicológico necessário para alcançar resultados de intervenção positivos é a experiência emocional. Segundo Walborn (1996), as emoções são o combustível da mudança. Os clientes chegam ao aconselhamento porque se sentem confusos, indecisos, com dificuldades em tomar decisões. Eles procuram alguém capaz de os ajudar e compreender. Sem compreensão emocional, os clientes continuam a sentir-se confusos e vazios, e sem motivação para a ação (Greenberg \& Safran, 1989). Providenciando um ambiente seguro, onde o cliente possa expressar as suas emoções, os psicólogos conseguem motivar o cliente para a mudança (Walborn, 1996).

Vários estudos com diferentes desenhos metodológicos (Karle, Hart, Gold, Maple, \& Hopper, 1976; Nichols \& Bierenbaum, 1978; Pierce, Nichols \& DuBrin, 1983), sustentam que as emoções constituem um elemento chave das variáveis de processo de aconselhamento psicológico. As pesquisas acerca da experiência dos clientes na intervenção psicológica apoiam amplamente a ideia que os clientes que se envolvem mais emocionalmente na intervenção, retiram mais benefício e têm mais sucesso na mesma (Luborsky, Chandler, Auerbach, Cohen \& Bachrach, 1971), quando comparados com os que têm um envolvimento baixo ou nulo.

Por fim, o quarto elemento comum e importante na eficácia da intervenção, envolve as expectativas dos clientes face ao aconselhamento. Diversos estudos (Friedman, 1963; Goldstein \& Shipman, 1961) demonstram que os clientes mais motivados, com expectativas realistas acerca dos acontecimentos do aconselhamento, e que esperam ser ajudados, beneficiam mais da intervenção, que os clientes com concepções erradas ou céticas acerca do seu processo (Kayano, 2009).

As investigações demonstram igualmente que socializar os clientes com a intervenção, explicando o que vai acontecer e o que é esperado obter, é benéfico para o sucesso da mesma. Com este procedimento, ajuda-se a eliminar concepções erradas sobre o processo de intervenção e a reduzir o abandono prematuro da intervenção (Piper, Debbane, Bienvenu, \& Garant, 1982; Truax \& Wargo, 1969).
O conhecimento destes resultados contribuiu para fortalecer a importância e dedicação atribuídas à pesquisa que relaciona o processo com os resultados da intervenção. Como refere Swanson (1995), paralelamente, o aconselhamento psicológico vocacional foi-se consolidando como sendo provido de processo e os investigadores foram reacendendo, cada vez mais, o seu interesse em investigar as variáveis desse mesmo processo.

\section{Modelos teóricos do processo de aconselhamento psicológico vocacional}

$\mathrm{Na}$ tentativa de compreender a complexidade do aconselhamento psicológico vocacional, vários autores têm proposto modelos de desenvolvimento deste processo, dos quais salientamos o Modelo Trifásico de Parsons, o Modelo Desenvolvimentista-Relacional de Taveira, e o Modelo de Adequação Cultural de Fouad e Bingham (Faria, 2008; Taveira, 2005).

O modelo de ajuda formulado por Parsons (1909), no livro Choosing a Vocation, é o primeiro modelo teórico de aconselhamento vocacional. Segundo este, o aconselhamento psicológico vocacional tem a configuração de uma psico-intervenção, uma vez que realça a importância do diagnóstico, da avaliação psicológica e da previsão, na exploração e na resolução de problemas de tomada de decisão e de ajustamento ou adaptação profissional (Taveira, 2005; Yost \& Corbishley, 1987).

O modelo trifásico de aconselhamento psicológico vocacional assume que o cliente apresenta um entre quatro problemas possíveis: (1) incapacidade para realizar uma escolha vocacional; (2) incerteza sobre a escolha vocacional realizada ou a realizar; (3) imprudência na escolha vocacional; e (4) discrepância entre interesses e atitudes vocacionais.

Os primeiros dois tipos de problema resultam da ausência ou insuficiência de informação pessoal e sobre o mundo do trabalho, ou de barreiras pessoais, como o medo de falhar, ou a insegurança relativa às suas competências. Estes fatores podem ser responsáveis pelo terceiro problema, a imprudência na escolha, em que a pessoa e o trabalho estão mal articulados. Por sua vez, o quarto problema, relaciona-se com a discrepância entre as competências do cliente e as competências exigidas num determinado trabalho.

Para qualquer um dos problemas mencionados, o aconselhamento psicológico vocacional tem como objetivo, ajudar o cliente a auto analisar-se, providenciar informação adequada acerca das ocupações e profissões e combinar os dados pessoais e do mundo ocupacional da pessoa, de modo a encontrar o ajuste perfeito entre ambos (Taveira, 2005; Yost \& Corbishley, 1987). Com efeito, segundo Parsons (1909), o processo de orientação vocacional é uma única escolha, num único momento da vida, definido a partir de três componentes: (1) a avaliação das características da pessoa - o psicólogo por meio de técnicas objetivas avalia o perfil da pessoa; (2) a avaliação profissional - análise e promoção das características de emprego para aquela pessoa; e (3) o estabelecimento de correspondências o mais perfeitas possíveis entre as características da pessoa e as características do mundo profissional. 
O modelo desenvolvimentista-relacional de Taveira (2001) segue uma abordagem desenvolvimentista centrada na pessoa, fundamentando-se na psicologia do desenvolvimento, para conceituar o apoio à resolução das questões de carreira em contexto de aconselhamento psicológico vocacional.

O modelo tem em conta o estatuto de desenvolvimento da carreira do cliente e adapta estratégias de aconselhamento a essas características, procurando promover tanto o desenvolvimento vertical, como horizontal (Taveira, 2001).

Advoga-se que é imprescindível aprovar o cliente na sua essência, isto é, como pensa, como sente e como age. Também é importante analisar como é que o cliente se situa em termos de desenvolvimento vocacional e apoiá-lo a progredir para níveis de desenvolvimento qualitativamente superiores. É um modelo caracterizado pela integração das dimensões humanista e técnica do aconselhamento psicológico vocacional. Neste sentido, a relação terapêutica constitui o âmago da intervenção (Taveira, 2001), desenrolando-se ao longo de quatro fases processuais: (1) iniciar; (2) explorar; (3) comprometer e; (4) finalizar (Tabela 1). Ao longo destas fases, o profissional de aconselhamento psicológico vocacional e cliente colaboram para clarificar os objetivos e a estratégia do cliente, mobilizar expectativas e promover processos cognitivo-afetivo-comportamentais sustentados pelo cliente.

Cada uma das fases envolve o prosseguimento de intenções e técnicas mais específicas, da parte do profissional do aconselhamento psicológico vocacional e expectativas quanto a reações mais específicas da parte do cliente (Gibson \& Mitchell, 1998; Spokane, 1991). Todas elas têm como base a aliança relacional entre psicólogo e cliente. Este modelo prevê, ainda, que o processo de intervenção envolve papéis e processos específicos, sendo, também, necessárias resoluções críticas de índole atitudinal, emocional, cognitiva e comportamental.

Fouad e Bingham (1995) propuseram um modelo de aconselhamento vocacional que evidencia a importância dos fatores culturais, baseado em cinco suposições teóricas: (1) a eficácia do aconselhamento psicológico vocacional toma lugar num dado contexto cultural, independentemente da etnia, considerando-se as questões de diversidade cultural dentro da cultura dominante; (2) as variáveis de ênfase cultural consideradas podem diferir, e o peso dado a essas variáveis pode variar de cultura para cultura; (3) os modelos teóricos de carreira estabelecidos não explicam adequadamente $o$ comportamento vocacional das minorias raciais e étnicas; (4) a importância de considerar a cultura nas avaliações vocacionais; e (5) a suposição que as diferenças culturais devem ser consideradas dentro de um contexto de diversidade ou pluralismo, e que os clientes devem ser atendidos no sentido da realização de escolhas culturais apropriadas (Sue \& Sue, 1990).

Nesta ordem de ideias, Fouad e Bingham (1995) propõem o Modelo de Adequação Cultural, constituído por nove passos: (1) a preparação para o aconselhamento; (2) o estabele-

Tabela 1. Fases do processo do aconselhamento vocacional (Taveira, 2001)

\begin{tabular}{|c|c|c|c|}
\hline \multirow{2}{*}{ Fases } & \multicolumn{2}{|l|}{ Psicólogo } & \multirow{2}{*}{$\begin{array}{l}\text { Cliente } \\
\text { Reações esperadas }\end{array}$} \\
\hline & Tarefas principais & Técnicas (foco) & \\
\hline \multirow[t]{5}{*}{ Iniciar } & Estabelecer uma aliança relacional & \multirow{5}{*}{$\begin{array}{l}\text { Oferta de estrutura } \\
\text { Aceitação do cliente }\end{array}$} & \\
\hline & Avaliar expectativas e interesses pela intervenção & & Alívio \\
\hline & Identificar necessidades e despistar psicopatologia & & Esperança \\
\hline & Focar nos modos como o cliente pensa e sente & & \\
\hline & Estabelecer um contrato intervenção & & \\
\hline \multirow[t]{7}{*}{ Explorar } & Compreender comportamentos e atitudes & \multirow{7}{*}{$\begin{array}{l}\text { Oferta de informação } \\
\text { Reflexão e clarificação } \\
\text { Imagética guiada } \\
\text { Testes e questionários } \\
\text { Apoio emocional } \\
\text { Reforço }\end{array}$} & \\
\hline & Atender às condições pessoais e do contexto & & Excitação \\
\hline & Atender a resistências & & Ansiedade \\
\hline & Desenvolver objetivos e planos & & Comportamentos aber- \\
\hline & Avaliar resultados da aliança relacional & & tos e encobertos \\
\hline & Ensaio de aspirações, fantasia, teste de hipóteses & & Compromisso \\
\hline & Identificar incongruências e zonas de conflito & & \\
\hline \multirow[t]{5}{*}{ Comprometer } & Ajudar a reduzir a ansiedade & \multirow{5}{*}{$\begin{array}{l}\text { Apoio à gestão da } \\
\text { ansiedade }\end{array}$} & \multirow{5}{*}{$\begin{array}{l}\text { Compromisso de } \\
\text { carreira }\end{array}$} \\
\hline & Ensinar ou reforçar o compromisso afectivo, cog- & & \\
\hline & nitivo e comportamental com opções exploradas & & \\
\hline & Envolver o cliente em testes da realidade & & \\
\hline & Avaliar resultados & & \\
\hline \multirow[t]{4}{*}{ Finalizar } & Antecipar passos e apoios necessários ao sucesso & \multirow{4}{*}{$\begin{array}{l}\text { Concluir o processo de } \\
\text { finalização da aliança } \\
\text { relacional }\end{array}$} & Satisfação \\
\hline & na concretização da solução/plano/opção desejada & & Certeza \\
\hline & Sensibilizar para necessidade de seguimento & & Independência face ao \\
\hline & deste processo & & psicólogo \\
\hline
\end{tabular}


cimento da aliança terapêutica; (3) a identificação dos temas/ questões da carreira a abordar; (4) a avaliação dos efeitos das variáveis culturais; (5) o estabelecimento de objetivos e de um processo do aconselhamento culturalmente apropriado; (6) a utilização de atividades culturalmente apropriadas; (7) a tomada de decisão; (8) a implementação dos planos do cliente; e (9) o seguimento.

O primeiro passo diz respeito à preparação para o aconselhamento pelo psicólogo. Fouad e Bingham (1995) arguiram que para que o aconselhamento psicológico vocacional com clientes de minorias raciais e étnicas seja eficaz, os psicólogos, antes do início efetivo do aconselhamento, deverão adquirir competências culturais transversais específicas. Assim, deverão começar por ter consciência, conhecimento e competências sobre a sua própria cultura e identidade racial e do impacto dessas nas suas atitudes e comportamentos profissionais. Em seguida, é importante que os psicólogos se familiarizem com alguma informação acerca de todos os grupos étnicos e culturais. É igualmente importante que os psicólogos clarifiquem, com o cliente, que as aspirações e expectativas acerca do mundo profissional deste, serão valorizadas e respeitadas, e que o psicólogo não as interpretará com base apenas na sua própria perspectiva, mas procurando ter em conta sobretudo a perspectiva do cliente (Armstrong, 2009; Arthur \& McMahon, 2005; Byars-Winston \& Foaud, 2006; Marshall, Young, \& Valach, 2007; Sue, Arrendondo, \& McDavis, 1992).

O segundo passo prende-se com o estabelecimento da aliança terapêutica, no contexto de uma relação culturalmente apropriada. As condições base para o estabelecimento desta relação são a empatia, o respeito, a atenção positiva, a genuinidade, a concentração e a proximidade. Contudo, as relações terapêuticas podem diferir transversalmente em função das culturas, sendo necessárias competências diferentes consoante aos diferentes grupos culturais.

O terceiro passo diz respeito à identificação dos temas ou questões de carreira. Fouad e Bingham (1995) sugerem que nesta fase é necessário dar uma atenção especial às dimensões emocionais, cognitivas, comportamentais e ambientais. Sugerem também que é relevante usar o modelo de Sue e Sue (1990) do locus de controle e de responsabilidade do cliente. Este modelo é constituído por quatro componentes: o locus interno de controle, o locus interno de responsabilidade, o locus externo de controle e o locus externo de responsabilidade.

O quarto passo é a avaliação do impacto das variáveis culturais. Fouad e Bingham (1995) sublinham a existência de cinco áreas de impacto das variáveis culturais: (1) fatores biológicos, (2) o gênero, (3) as expectativas da família relativamente ao futuro profissional dos filhos, o estilo parental da família, a existência ou não de um chefe familiar, (4) o grupo racial-étnico e, (5) a cultura dominante (Arthur \& McMahon, 2005).

O quinto passo do aconselhamento diz respeito ao estabelecimento de objetivos e do processo de aconselhamento de modo culturalmente apropriado. Os objetivos deverão centrar-se na clarificação dos interesses do cliente bem como na tomada de decisão de carreira.

O sexto passo corresponde à intervenção propriamente dita, com recurso a atividades de aconselhamento culturalmente apropriadas. Quando indicado, e possível, a inter- venção deverá realizar-se na língua nativa do cliente, assim como, dependendo da identidade racial do cliente, deverá ser efetuada por um psicólogo da mesma raça ou etnia (Byars-Winston \& Foaud, 2006).

O sétimo passo é a tomada de decisão. Nesta fase os clientes são chamados a realizar decisões acerca dos papéis de trabalho, da saliência dos diferentes papéis de vida (família, lazer, trabalho) e da necessidade ou não de mais informação para a tomada de decisão de carreira. Nem todos os clientes encaram a decisão de um modo racional e linear. Alguns clientes podem fazer planos, realizá-los e sair do processo. Outros clientes têm necessidade de voltar atrás no processo de aconselhamento, para clarificar as suas questões ou preocupações de carreira. Neste caso, é importante analisar com o cliente se o plano de carreira é congruente com as suas visões do mundo (Armstrong, 2009; Arthur \& McMahon 2005; McMahon, 2005).

O oitavo passo diz respeito à implementação dos planos do cliente. Nesta fase os clientes tomam decisões de carreira. É esperado que o psicólogo acredite que os planos do cliente satisfazem os requisitos do seu ponto de vista do mundo e que ajude o cliente a prever algumas consequências das decisões tomadas.

Por último, o nono passo corresponde ao seguimento. $\mathrm{O}$ seguimento consiste em encorajar o cliente a regressar ao aconselhamento sempre que necessário, garantindo que isso não é entendido como um fracasso no processo. O cliente pode voltar atrás no processo para clarificar os seus resultados de carreira, reinicinado assim o passo dois - identificação dos resultados de carreira (Fouad \& Bingham, 1995; Subich \& Simonson, 2001).

Com o desenho de modelos teóricos do processo de aconselhamento psicológico vocacional, providenciou-se uma perspectiva sobre o estado da arte do estudo desta problemática, aumentando-se a compreensão sobre como é que a aconselhamento psicológico vocacional deve ser conduzido (McMahon, 2005; Swanson, 1995; Whiston e cols., 2005). O processo do aconselhamento psicológico vocacional foi referido como tudo o que acontece durante as sessões de aconselhamento, em termos de comportamento do psicólogo e do cliente separadamente, e entre ambos (Heppner \& Jordan, 2007; Hill \& Willams, 2000). Com efeito, inclui as intenções do psicólogo (Hill \& O'Grady, 1985), assim como as reacções do cliente (Hill, Helms, Spielgel, \& Tichenor, 1988), ou seja, os pensamentos abertos e encobertos, sentimentos e comportamentos de ambos.

Hill e Williams (2000) caracterizaram o processo do aconselhamento psicológico vocacional por meio dos conceitos de variáveis de entrada, acontecimentos extra-aconselhamento e resultados do aconselhamento. As variáveis de entrada envolvem as características do cliente (personalidade, fatores demográficos, expectativas face à intervenção) e do psicólogo (personalidade, fatores demográficos, orientações teóricas) e características do espaço físico (aspecto físico da sala de aconselhamento, tutela pública ou privada dos serviços). Os acontecimentos extra-aconselhamento ocorrem fora das sessões e podem facilitar ou impedir o desenrolar do processo do aconselhamento. Por sua vez, os resultados envolvem quer efeitos imediatos, por exemplo, de uma sessão de aconselhamento, (respostas cognitivas do cliente a ações específicas), 
quer efeitos intermediários, se as mudanças ocorrem como resultado de eventos terapêuticos ou de uma sessão, ou ainda efeitos longínquos, se as respostas ocorrem como resultado de todo o processo de intervenção (Heppner \& Heppner, 2004; Heppner \& Jordan, 2007; Kayano, 2009; Swanson, 1995).

\section{Estudos empíricos do processo do aconselhamento psicológico vocacional}

Diversas investigações e metodologias têm sido ensaiadas empiricamente na tentativa de compreender a complexidade do processo de aconselhamento psicológico vocacional (Faria, 2008). Por exemplo, Bacorn e Dixon (1984), com o objetivo de testar o efeito do toque físico no aconselhamento psicológico vocacional, selecionaram mulheres voluntárias, com dois tipos de diagnóstico, depressão e indecisão de carreira, e submeteram-nas a duas condições: toque e não toque. As sessões individuais tiveram a duração de trinta minutos e foram conduzidas por cinco psicólogos homens. Não foram observadas diferenças quanto ao problema apresentado, quanto à presença ou ausência de toque, características da relação, ou vontade de voltar a uma segunda entrevista. Contudo, as participantes cuja entrevista se referia a questões de carreira ficaram significativamente mais confortáveis com o toque, do que as participantes com diagnóstico de depressão. O maior nível de conforto aparente, não surgiu, porém, refletido nas percepções das clientes acerca do psicólogo.

Lillard (1991), com o objetivo de examinar as diferenças no estilo de discurso do psicólogo - ensaio ou absoluto - convidou sujeitos a visualizarem um de dois vídeos de aconselhamento psicológico, de oito minutos cada. Os vídeos descreviam uma cliente mulher e um psicólogo homem, a discutir vários mitos de carreira e caminhos para "vencê-los". $\mathrm{O}$ vídeo relativo ao estilo ensaio recorria ao uso de frases interrogativas, enquanto o vídeo relativo ao estilo absoluto recorria a frases declarativas. Não foram observadas diferenças entre as condições de estilo de discurso nas percepções dos clientes. Os mitos acerca da carreira foram reduzidos em ambas as condições.

Por sua vez, Miller (1992), com o objetivo de testar o efeito da tomada de notas no aconselhamento psicológico vocacional, expôs sujeitos a dois vídeos de 14 minutos cada, sendo que num deles o psicólogo tomava notas breves cinco vezes durante a sessão, enquanto no outro não tirava qualquer tipo de notas. Não se registaram diferenças na avaliação dos clientes das destrezas do psicólogo, nem na atração ou merecimento de confiança deste, em função da tomada de notas. Os participantes indicaram maior vontade para ver o psicólogo na condição de não tomada de notas.

Com o objetivo de analisar as expectativas do aconselhamento psicológico vocacional, Galassi, Crace, Martin, James e Wallace (1992) questionaram os clientes acerca daquilo que eles preferiam e antecipavam do aconselhamento psicológico vocacional, por meio de 15 perguntas de resposta semi-aberta, na primeira sessão de aconselhamento. Os resultados demonstram que os clientes: (a) preferem e antecipam sessões de aconselhamento que não sejam sempre iguais; (b) têm uma ideia clara acerca das suas preferên- cias; (c) consideram que o aconselhamento psicológico vocacional deveria ter três sessões, os resultados deveriam ser de direcão ou confirmação da escolha e, os testes têm um importante papel; (d) preferem um aconselhamento que foque planos específicos de carreira e de tomada de decisão e, a exploração do self e do meio; (e) preferem um psicólogo ativo que dê conselhos e exercícios de exploração de carreira entre as sessões de aconselhamento; (f) tinham menos claro o que antecipavam e gostariam que ocorresse no aconselhamento psicológico vocacional. Além disso, tendo em conta as discrepâncias entre preferências e antecipações dos participantes face ao aconselhamento, os resultados deste estudo sugerem que os clientes antecipavam menos concretizações da parte do psicólogo do que as que desejavam que ocorressem.

Heppner, Multon, Gysbers, Ellis, e Zook (1998) estudaram a relação entre a auto-eficácia do psicólogo no aconselhamento psicológico vocacional e medidas de processo e resultado nesse mesmo contexto. Para tal, analisaram a resposta de 24 estagiários de psicologia, que atenderam 55 clientes entre três e 12 sessões individuais, tendo aplicado a medida Career Counseling Self-Efficacy Scale desenvolvida por O'Brien, Heppner, Flores e Bikos (1997), antes e depois da intervenção com cada cliente. Os clientes preencheram várias medidas de resultado. Os resultados demonstram que a auto-eficácia do psicólogo aumentou do pré para o pós teste e que os resultados dos clientes aumentam significativamente do pré para o pós teste. No final da intervenção, verifica-se um crescimento significativo da aliança de trabalho, da capacidade para definir objetivos e decidir. $\mathrm{O}$ aumento dos valores nas variáveis de processo e a diminuição dos valores nas variáveis de resultados parecem não ter qualquer relação com o sentimento de auto-eficácia do psicólogo.

Multon, Ellis-Kalton, Heppner e Gysbers (2003), por sua vez, com o objetivo de discutir o uso de diferentes tipos de resposta do psicólogo no aconselhamento vocacional, analisaram as respostas de 19 psicólogos em formação e de 26 clientes, em 78 sessões de aconselhamento psicológico vocacional. Do total, $79 \%$ das respostas dos psicólogos foram de tipo informação, paráfrase ou questões fechadas. Nenhuma das respostas dos psicólogos se relacionou significativamente com a relação terapêutica, embora a auto-revelação dos psicólogos registrasse uma correlação negativa significativa com a relação terapêutica. O tipo de auto-revelação usado era na maioria das vezes irrelevante.

Em síntese, os diferentes estudos demonstram a existência de preferências dos clientes por estilos específicos de aliança terapêutica e estratégias de acção (Galassi e cols., 1992), apesar de se verificar, ao mesmo tempo, que variações nas respostas verbais dos psicólogos afetam pouco ou mesmo nada as percepções dos clientes acerca da intervenção (Lillard, 1991) e que comportamentos como tirarem notas e o toque físico poderão, pelo contrário, ter um impacto nessas mesmas percepções (Bacorn \& Dixon, 1984; Miller, 1992). As variáveis estudadas ilustram a complexidade do processo de intervenção vocacional, até hoje, no entanto, pouco explorada. Sua investigação parece determinante para a estruturação de boas práticas baseada no suporte empírico e para o reforço da sua unicidade. 


\section{Sugestões para a investigação futura}

Quando comparada a investigação dos processos de aconselhamento psicológico pessoal e vocacional verifica-se a existência de algumas lacunas neste último. Tendo em conta a evidência empírica apresentada sobre os fatores comuns de eficácia (Anderson \& Niles, 1995; Walborn, 1996) e o conhecimento sobre os aspectos mais úteis ao processo de aconselhamento psicológico pessoal (Hill \& Williams, 2000), autores como Heppner e Heppner (2003) consideram que se pode alargar os resultados da pesquisa do aconselhamento psicológico pessoal ao aconselhamento vocacional.

De acordo com esta ideia, uma das primeiras linha de investigação a seguir no estudo do processo de aconselhamento psicológico vocacional deve ser a identificação dos comportamentos do cliente e do psicólogo que conduzem à formação e desenvolvimento da relação terapêutica (Multon, Heppner, Gysbers, Zook \& Ellis-Kalton, 2001).

Uma segunda linha consiste no estudo das técnicas utilizadas pelo psicólogo para conduzir o processo de intervenção. No aconselhamento psicológico pessoal, Hill e Williams (2000) identificaram cinco técnicas específicas do psicólogo que facilitam o processo terapêutico: a interpretação, a confrontação, a auto-revelação, a intenção paradoxal e as estratégias para incentivar a ação do cliente. Segundo Heppner e Heppner (2003) essas técnicas poderiam provocar os mesmos efeitos no aconselhamento psicológico vocacional.

Uma terceira linha de investigação poderá ser relacionar a visão do aconselhamento psicológico vocacional com os processos de aprendizagem. Strupp (1986) apurou que o aconselhamento psicológico pessoal era, primeiramente, uma combinação de duas variáveis: a aprendizagem e a reaprendizagem. Assim, definiu o psicólogo como alguém que poderia promover a aprendizagem do cliente. Subsequentemente, com o passar dos anos, os investigadores identificaram consistentemente que variáveis de resultado refletiam a aprendizagem do cliente no aconselhamento psicológico pessoal, e que variáveis de processo prediziam esses resultados. No caso do aconselhamento psicológico vocacional, o cliente pode aprender, por exemplo, mensagens novas sobre o que são as escolhas de carreira, sobre os papéis de vida considerados apropriados ao seu sexo de pertença, ou ainda, sobre novas competências. Similarmente, os clientes podem necessitar de realizar reaprendizagens acerca de quem são realmente.

Uma quarta linha de investigação prende-se com o estudo dos subtipos de clientes de carreira. Estudos analíticos de agrupamentos de clientes, conduzidos com estudantes indecisos (Larson \& Majors, 1998), demonstram que nem todos os clientes do aconselhamento psicológico vocacional são iguais. Brown e Krane (2000) consideram, a este propósito, que há três tipos de clientes vocacionais: (a) os clientes que necessitam apenas de informação ocupacional adicional e ajuda na exploração ocupacional; (b) os clientes, cujos problemas de carreira-escolha se relacionam primeiramente com a ansiedade; e (c) os clientes severamente afetados que apresentam uma constelação de problemas. Assim, parece promissor examinar como o processo de aconselhamento fica afetado por estes subtipos de clientes.

Por último, uma outra linha de investigação a prosseguir, relaciona-se com as perspectivas culturais dos clientes e dos psicólogos (Cook, Heppner \& O’Brien, 2004; Cook, Hepp- ner \& O’Brien, 2005). Apesar da existência de um modelo de organização do processo da aconselhamento psicológico vocacional que tem em consideração as variáveis culturais (Fouad \& Bingham, 1995; Marshall e cols., 2007) assiste-se, ainda, a uma relativa homogeneização dos clientes da carreira, verificando-se uma falta da informação sobre como diferentes perspectivas culturais do psicólogo e do cliente afetam o processo e os resultados do aconselhamento psicológico vocacional. Há evidência na literatura do aconselhamento psicológico pessoal para concluir que os clientes de minorias raciais e étnicas tendem a terminar prematuramente a intervenção, e numa taxa maior que os clientes brancos (Sue \& Sue, 1990). Daí a importância de aprofundar estes mecanismos culturais, também, na interação terapêutica do aconselhamento vocacional (Armstrong, 2009; Arthur \& McMahon, 2005).

\section{Referências}

Anderson, W. P., \& Niles, S. G. (1995). Career and personal concerns expressed by career counseling clients. Career Development Quarterly, 43, 240-245.

Armstrong, L. (2009). Multicultural career counseling: Toward a new paradigm for the career development of women and ethnic minorities. River Academic Journal, 5(1), 1-4.

Arthur, N., \& McMahon, M. (2005). Multicultural career counseling: Theoretical applications of the systems theory framework. Career Development Quarterly, 53(3), 208- 222.

Bacorn, C. N., \& Dixon, D. N. (1984). The effects of touch on depressed and vocationally undecided clients. Journal of Counseling Psychology, 31, 488-496.

Bedi, R. P. (2004). The therapeutic alliance and the career counseling/personal counseling interface. Journal of Employment Counseling, 41, 126-135.

Bergman, D. L. (1951). Counseling method and client responses. Journal of Consulting Psychology, 15, 216-224.

Blustein, D. L., \& Spengler, P. M. (1995). Personal adjustment: Career counselling and psychotherapy. Em Walsh, W., \& Osipow, S. (Eds.), Handbook of Vocational Psychology: Theory, Research, and practice (2th ed., pp, 295-328). New Jersey: Lawrence Erlbaum Associates, Publishers.

Brown, S. D., \& Krane, N. E. (2000). Four (or five) sessions and a cloud of dust: Old assumptions and new observations about career counseling. Em S. Brown \& R. Lent (Eds.), Handbook of Counseling Psychology (3th ed., pp. 740-749). New York: John Wiley \& Sons, Inc.

Byars-Winston, A. M., \& Foaud, N. A., (2006). Metacognition and multicultural competence: Expanding the culturally appropriate career counseling model. Career Development Quarterly. 54(3), 187-202.

Cook, E. P., Heppner, M. J., \& O’Brien, K. M. (2004). An ecological model of career development. Em R. K. Conyne \& E. P. Cook (Eds.), Ecological counseling: An innovative approach to conceptualizing person-environment interaction. American Counseling Association Press.

Cook, E. P., Heppner, M. J., \& O’Brien, K. M., (2005). Multicultural and gender influences in women's career development: An ecological perspective. Journal of Multicultural Counseling and Development, 33, 165-179 
Faria, L. F. (2008). A eficácia da aconselhamento psicológica vocacional de jovens: Estudo do impacto de uma intervenção. Tese de Doutorado, Braga: Universidade do Minho.

Fouad, N., \& Bingham, R. (1995). Career counseling with racial and minorities. Em W. B. Walsh \& S. Osipow (Eds.), Handbook of vocational psychology: Theory, research and practice (pp. 331-365). Mahwah, NJ: Lawrence Erlbaum Associates.

Friedman, H. J. (1963). Patient-expectancy and symptom reduction. Archives of Therapy, 21, 189-196.

Galassi, J., Crase, R.; Martin, G. James, R., \& Wallace, R. (1992). Client preferences and anticipation in career counseling: A Preliminary Investigation. Journal of Counseling Psychology, $39,46-55$.

Gelso, C. J., \& Carter, J. A. (1985). The relationship in counseling and psychotherapy: Components, consequences, and theoretical antecedents. Counselling Psychologist, 13, 155-243.

Gelso, C. J., \& Cárter, J. A. (1994). Components of the psychotherapy relationship: Their interaction and unfolding during treatment. Journal of Counseling Psychology, 41, 296-306.

Gibson, R. C., \& Mitchell, M. H. (1998). Introduction to counseling and guidance. ( $5^{\text {th }}$ ed., pp. 122-177). New Jersey: Prentice Hall.

Gold, P. B., Kivlighan, D. M., Kerr, A. E., \& Kramer, L. A. (1993). The structure of students perceptions of impactful, helpful events in career exploration classes. Journal of Career Assessment, 1, 145-161.

Goldman, L. (1976). A revolution in counseling research. Journal of Counseling Psychology, 23(6), 543-552.

Goldstein, A. P., \& Shipman, W. G. (1961). Patient expectancies, symptom reduction and aspects of initial psychotherapeutic interview. Journal of Clinical Psychology, 17, 129-133.

Greenberg, L. S., \& Safran, J. D. (1989). Emotion in psychotherapy. American Psychologist, 44, 19-29.

Gunderson, J. G., Najavits, L. M., Leonhard, C., Sullivan, C. N., $\&$ Sabo, A. S. (1997). Ontogeny of the therapeutic alliance in borderline patients. Psychiatry Research, 7, 301-309.

Gurman, A. S. (1977). The patient's perception of the therapeutic relationship. Em A. S. Gurman \& A. M. Razin (Eds.), Effective psychotherapy: A handbook of research (pp. 503-543). NY: Pergamon Press.

Gysbers, N. C. (2004). Life career development: A needed perspective for all counseling. Em G. R. Walz \& R. Yep (Eds), Vistas: Perspectives on counseling 2004, CD Rom, American Counseling Association and Counseling Outfitters/CAPS Press.

Gysbers, N. C., Heppner, M. J., \& Johnston, J. A. (2003). Career counseling: Process, issues, and techniques (2th ed.). Boston, MA: Allyn and Bacon.

Hartley, D. E., \& Strupp, H. H. (1983). The therapeutic alliance: Its relationship to outcome in brief psychotherapy. Em J. Masling (Ed.), Empirical studies in analytic theories (pp. 1-37). Hillsdale, NJ: Erlbaum

Heppner, M. J., \& Hendricks, F. (1995). A process and outcome study examining career indecision and indecisiveness. Journal of Counseling and Development, 73, 426-437.

Heppner, M. J., \& Heppner, P. P. (2003). Identifying process variables in career counseling: A research agenda. Journal of Vocational Behavior, 62, 429-452.

Heppner, M. J., \& Heppner, P. P. (2004). Career counseling. Em C. Spielberger (Ed.), The encyclopedia of applied psychology. San Diego, CA: Academic Press.
Heppner, M. J., \& Heppner, P. P. (2007). Quali aspetti del processo di career counseling conducono a risultati efficaci. Giornale Italiano di Psicologia dell'Orientamento, 7(3),3-7.

Heppner M. J., \& Jordan, S. (2007). Career process and outcome: The encyclopedia of counseling. Thousand Oaks, CA: Sage.

Heppner, M. J., Multon, K. D., Gysbers, N. C., Ellis, C. A., \& Zook, C. E. (1998). The relationship of trainee self-efficacy to the process and outcome of career counseling. Journal of Counseling Psychology, 45(4), 393-402.

Heppner, P. P., \& Heppner, P. P. (2007). Il problem solving nel processo di career counseling. Giornale Italiano di Psicologia dell'Orientamento, 7(3), 9-13.

Hill, C. E., Carter, J. A., \& O'Farrell, M. K. (1983). A case study of the process and outcome of time-limited counselling. Journal of Counseling Psychology, 30(1), 3-18.

Hill, C. E. Helms, J. E., Spielgel, S. B., \& Tichenor, V. (1988). Development of a system for categorizing client reactions to therapist interventions. Journal of Counseling Psychology, $35,27-36$.

Hill, C. E., \& O'Grady, K. E. (1985). A list of therapist intentions illustrated in case study and with therapists of varying theoretical orientations. Journal of Counseling Psychology, 32, 3-22.

Hill, C., \& Williams, E. (2000). The process of individual therapy. Em S. Brown \& R. Lent (Eds.), Handbook of counseling psychology. (3th ed., pp.670-710). NY: John Wiley \& Sons, Inc.

Holland, J. L., Magoon, T. M., \& Spokane, A. R. (1981). Counseling psychology: Career interventions, research, and theory. Annual Review of Psychology, 32, 279-305.

Horvath, A. O., \& Luborsky, L. (1993). The role of the therapeutic alliance in psychotherapy. Journal of Consulting and Clinical Psychology, 61(4), 561-573.

Karle, W., Hart, R., Gold, J., Maple, S., \& Hopper, M. (1976). The maintenance of psychophysiological changes in feeling therapy. Psychological Reports, 39, 1143-1147.

Kayano, J. (2009). Conscientization of a carrer counseling process: Becoming aware of one's career counseling process. Japan Labor Review, 6(4), 135-156.

Kivlighan, D., \& Shaughnessy, P. (1995). Analysis of the development of the working alliance using hierarchical linear modeling. Journal of Counseling Psychology, 42(3), 338-349.

Krischner, T., Hoffman, M., \& Hill, C. (1994). Case study of the process and outcomes of career counseling. Journal of Counseling Psychology, 41, 216-226.

Lewis, J. (2001). Career and personal counseling: Comparing process and outcome. Journal of Employment Counseling, $38,82-90$.

Luborsky, L., Chandler, M., Auerbach, A. H., Cohen, J., \& Bachrach, H. M. (1971). Factors influencing the outcome of psychotherapy: A review of quantitative research. Psychological Bulletin, 75, 145-85.

Larson, L. M., \& Majors, M. S. (1998). Applications of the coping with career indecision instrument with adolescents. Journal of Career Assessment, 6, 163-179.

Lillard, M. N. (1991). Career counseling from a social influence perspective. Effects of interpretation style with career indecisive clients. (Unpublished doctoral dissertation). Memphis State University, Memphis. 
Malan, D. H. (1973). The outcome problem in psychotherapy research: A historical review. Archives of General Psychiatry, 29, 719-729.

Marshall, S. K., Young, R. A., \& Valach, L. (2007). Making career theories more culturally sensitive: Implications for counseling. The Career Development Quarterly, 56(1); 4-18

McMahon, M. (2005). Career counseling: Applying the systems theory framework of career development. Journal of Employment Counseling. 42(1), 29-39.

Miller, M. (1992). Effects of note-taking on perceived counselor social influence during a career counseling session. Journal of counseling Psychology. 39, 317-320.

Multon, K. D., Ellis-Kalton, C. A., Heppner, M. J., \& Gysbers, N. $\mathrm{C}$ (2003). The relationship between counselor verbal response modes and the working alliance in career counseling. The Career Development Quarterly, 51(3), 259-273.

Multon, K. D., Heppner, M. J., Gysbers, N. C., Zook, C. E., \& Ellis-Kalton, C. (2001). Client psychological distress: An important factor in career counseling. The Career Development Quarterly, 49, 324-335

Multon, K. D., Wood, R., Heppner, M. J., \& Gysbers, N. C. (2007). A cluster analytic investigation of subtypes of adult career counseling clients: Toward a taxonomy of career problems. Journal of Career Assessment, 15, 66-86.

Nichols, M. P., \& Bierenbaum, H. (1978). Success of cathartic therapy as a function of patient variables. Journal of Consulting and Clinical Psychology, 34, 776-778.

O’Brien K. M., Heppner M. J., Flores L. Y. \& Bikos L. H. (1997). The career counseling self-efficacy scale: Instrument development and training applications. Journal of Counseling Psychology, 44, 20-31

Orlinsky, D. E., \& Howard, K. I. (1986). Process and outcome in psychotherapy. Em S. L. Garfield \& A. E. Bergin (Eds.), Handbook of psychotherapy and behavior change (3th ed., pp. 311-384). NY: Wiley.

Parsons, F. (1909). Choosing a Vocation. Boston: Houghton-Mifflin.

Patterson, C. (1974). Relationship counseling and psychotherapy. NY: Harper \& Row.

Pierce, R. A., Nichols, M. P., \& DuBrin, J. R. (1983). Emotional expression in psychotherapy. NY: Gardner Press.

Piper, W. E., Debbane, E. G., Bienvenu, J. P., \& Garant, J. (1982). A study of group pretraining for group psychotherapy. International Journal of Group Psychotherapy, 32(3), 309-325.

Porter, E. H, Jr. (1943). The development and evaluation of a measure of counseling interview procedures. Educational and Psychological Measurement, 3, 105-126.

Robinson, F. P. (1950). Principles and procedures in student counseling. New York: Harper \& Row.
Spokane, A. R. (1991). Career intervention. Englewood Cliffs, NJ: Prentice Hall.

Strupp, H. H. (1986). Psychotherapy research, practice, and public policy (How to avoid dead ends). American Psychologist, 41(2), 120-130.

Subich, L. M., \& Simons, K. (2001). Career counseling: The evolution of theory. Em F. T. L. Leong \& A. Barack (Eds.), Contemporary models in vocational psychology. A volume in honor of Samuel H. Osipow (pp. 257-278). Mahwah, NJ: Lawrence Erlbaum.

Sue, D. W., \& Sue, D. (1990). Counseling the culturally different: Theory and practice (2th ed.). NY: Wiley.

Sue, D. W., Arrendondo, P., \& McDavis, R. J. (1992). Multicultural counseling competencies and standards: A call to the profession. Journal of Multicultural Counseling and Development, 20, 64-88.

Swanson, J. (1995). The process and outcomes of career counseling. Em S. H. Osipow \& W. B. Walsh (Eds.). Handbook of vocational psychology, theory research, and practice. (2th ed., pp. 217-260). Mahwah, NJ: Lawrence Erlbaum.

Taveira, M. C. (2001). O modelo de intervenção vocacional por programas. Lisboa: Casa Pia de Lisboa.

Taveira, M. C. (2005). Comportamento e desenvolvimento vocacional da adolescência. Em M. C. Taveira (Ed.), Psicologia escolar. Uma proposta científico-pedagógica (pp.143-178). Coimbra: Quarteto.

Truax, C. B., \& Wargo, D. G. (1969). Effects of vicarious therapy pretraining and alternative sessions on outcome in group psychotherapy with outpatients. Journal of Consulting and Clinical Psychology, 33, 440-447.

Walborn, F. (1996). Process variables: Four common elements of counseling and psychotherapy. Pacific Grove: Brooks/Cole Publishing Company.

Whiston, S., Lindeman, D., Rahardja, D., \& Reed, J. (2005). Career counseling process: A qualitative analysis of experts' cases. Journal of Career Assessment, 13, 169-187.

Yost, E. B., \& Corbishley, M. A. (1987). Career counseling: A psychological approach (pp. 21-23). San Francisco, CA: Jossey-Bass. 